\title{
State-dependent Data Queuing in Shared-resource Networked Control Systems
}

\author{
Mohammad H. Mamduhi ${ }^{1,2}$, John S. Baras ${ }^{1,2,3}$, Karl Henrik Johansson ${ }^{1}$, and Sandra Hirche ${ }^{2}$
}

\begin{abstract}
In the design of shared resource networked control systems (NCSs), resource managers play an important role to appropriately allocate limited resources across the distributed system. They are often used to fairly distribute the limited bandwidth among the medium-sharing entities at the expense of delaying or discarding unnecessary data samples. Considering the rapidly growing volume of information being exchanged, a relevant scenario for efficient resource management is statedependent data buffering via network queues. In this paper, we propose state-dependent data buffering for shared-resource NCSs, such that the buffer state, i.e. queue length, can be controlled depending on the real-time conditions of both the control systems and the communication network. We consider that the transmission decisions at the sensor sides are taken by event-based schedulers, and those data eventually sent for transmission are queued and processed depending on the available communication resource. We derive sufficient conditions under which the NCS with the proposed cross-layer transmission scheme is stable in almost sure mean-square sense. Moreover, we show performance improvements resulting from our proposed design in comparison with its state-independent counterpart.
\end{abstract}

\section{INTRODUCTION}

In the design of shared resource multi-loop networked control systems (NCSs), intelligent management of limited or costly communication resources is a crucial aspect to avoid excessive cost, as well as distributing the available resources fairly among the medium-sharing entities. It is essential to provide access to fast and low-error communication infrastructure to facilitate information exchange between distributed parts of a networked control system. This, however, imposes high communication and computation costs as well as network-induced phenomena such as delay, congestion, and data loss, thus, urges to reconsider the employment of traditional time-triggered sampling techniques [1]. Various approaches, such as event-based sampling, scheduling, data buffering, and network queuing, are developed to coordinate data exchange in an NCS leading to, first, the reduction of

\footnotetext{
${ }^{1}$ M. H. Mamduhi, J. S. Baras, and K. H. Johansson are with the Department of Automatic Control, The Royal Institute of Technology, Stockholm, Sweden, \{mamduhi, kallej\}@kth. se

${ }^{2}$ M. H. Mamduhi, J. S. Baras, and S. Hirche are with the Chair of Information-Oriented Control, Technical University of Munich, Munich, Germany, \{mh.mamduhi, hirche\}atum.de

${ }^{3} \mathrm{~J}$. S. Baras is with the Department of Electrical \& Computer Engineering, Institute for Systems Research, University of Maryland, Maryland, USA, baras @umd.edu

This work is jointly supported by the German Research Foundation (DFG) within the Priority Program SPP 1914 "Cyber-Physical Networking", the Knut and Alice Wallenberg Foundation, the Swedish Strategic Research Foundation, the Swedish Research Council, and DARPA through ARO grant W911NF1410384, and by ONR grant N00014-17-1-2622.
}

communication and computational costs by restricting excessive data sampling, and, second, intelligent coordination of transmissions among the distributed users to avoid excessive traffic and latency. Despite the positive aspects, these approaches often induce delay in some parts of the NCS leading to error propagation, deteriorating control performance, and even instability. Hence, these sampling policies need to be carefully synthesized to preserve stability and provide the required quality-of-control (QoC) guarantees [2], [3].

Event-based sampling was introduced in the early 2000s as a beneficial design framework to perform sampling of signals based on urgency metrics, e.g. an action is executed only when some pre-defined events are triggered [4]. This idea is further developed as an online technique capable of significantly reducing the sampling rate while preserving the required QoC [5]-[9]. The mentioned works address sporadic sampling of NCSs governed by real-time conditions of the medium-sharing control systems or the communication medium. Having NCSs as the integration of multiple control systems supported by a communication network, crosslayer scheduling attracted more attention. The reason is that scheduling induces delay and affects NCS stability and QoC, hence, scheduling approaches that take into account real-time conditions of control systems become popular [10]-[14].

Data queuing is a traditional resource management technique aiming to maximize quality-of-service (QoS), expressed often in the form of bandwidth or throughput requirements [15], [16]. The idea has gone through various modifications, e.g. accounting for congestion by back-pressure algorithms, or collision avoidance via back-off mechanisms, to adjust to the new communication trends [17]-[19]. The mentioned works consider queuing data that arrive from asynchronous nodes across the network, and then designing buffer discharge mechanisms, such as first-in-first-out (FIFO) or last-in-first-out (LIFO), to release data to the corresponding end-nodes. The queue models used in these works are static, and service is independent of the dynamics of sending and receiving nodes. In event-triggered NCSs wherein control systems have similar sampling periods, multiple requests may arrive at the buffer simultaneously, and in addition, arrival rates are state-dependent as sub-systems decide in real-time either to request for a transmission or not. This leads to coupling between the buffer dynamics and systems dynamics. To the best of the authors' knowledge, data queuing in event-triggered NCSs has not been fundamentally addressed, though a few exceptions exist, e.g., [20], [21].

The main contribution of this paper is proposing a crosslayer queue-based transmission mechanism that combines 
two approaches of event-triggered sampling and data queuing for NCSs consisting of multiple linear time-invariant (LTI) sub-systems. We take the most basic scenario of thresholdbased event-triggers and FIFO queues to present our results. The results however are extendable to more complicated models such as priority queues and LIFO buffers. The decisions on transmission requests are locally taken by eventtriggered schedulers. Those data are queued and processed depending on the available bandwidth. We demonstrate that the proposed state-dependent data queuing method is capable of maintaining the overall NCS stability in meansquare sense, while significantly reducing the total sampling instances without degrading control performance.

In the reminder of this paper, we state the problem of interest in Section II. Section III presents the state-dependent data queuing model. Stability results are provided in Section IV. Numerical illustrations are afterwards shown in Section V.

\section{PROBLEM STATEMENT}

We consider an NCS consisting of $N$ heterogeneous LTI control loops that are exchanging information through a shared communication network. Each individual loop consists of a linear stochastic plant $\mathcal{P}_{i}$ and a linear feedback controller $\mathcal{C}_{i}, i \in\{1, \ldots, N\}$. The plant $\mathcal{P}_{i}$ is modeled according to the following linear stochastic difference equation

$$
x_{k+1}^{i}=A_{i} x_{k}^{i}+B_{i} u_{k}^{i}+w_{k}^{i}
$$

where $x_{k}^{i} \in \mathbb{R}^{n_{i}}$ represents the system state, $u_{k}^{i} \in \mathbb{R}^{m_{i}}$ denotes the control signal executed at time $k$, and $w_{k}^{i} \in \mathbb{R}^{n_{i}}$ is the $i^{\text {th }}$ sub-system's exogenous disturbance. The constant matrices $A_{i} \in \mathbb{R}^{n_{i} \times n_{i}}$, and $B_{i} \in \mathbb{R}^{n_{i} \times m_{i}}$ describe the system matrix, and input matrix of sub-system $i$. The disturbance is assumed to be a random sequence with independent and identically distributed (i.i.d.) realizations $w_{k}^{i} \sim \mathcal{N}\left(0, W_{i}\right)$, with $W_{i} \succ 0$ denoting the variance of the normal distribution. The initial state $x_{0}^{i}$ is also randomly chosen from an arbitrary finitemoment distribution and is independent of the disturbance sequence. For simplicity, we assume that the sensor measurements are perfect copies of the system states, i.e., the output matrix is unity, and no measurement noise exists. Moreover, it is assumed that each pair $\left(A_{i}, B_{i}\right)$ is locally controllable.

The communication channel which supports the information exchange between the plants and the controllers, is assumed to have resource limitations, i.e., the bandwidth is not sufficient for all sub-systems to transmit simultaneously. In fact, the data packets sent from the individual sub-systems are queued in a single buffer and a transmission is completed when the packet is within the range of the departing bandwidth. This may take multiple time-steps from the time of the transmission request, hence, state information is received by the corresponding controller with buffer-induced delay. To take this effect into account, we denote the queue length at a time-step $k$ by $l_{k}$, with the initial length $l_{0}<\infty$. For technical reasons, we assume that the buffer provides infinite capacity, hence, every data packet sent for transmission will be added to the waiting queue. The buffer input and output

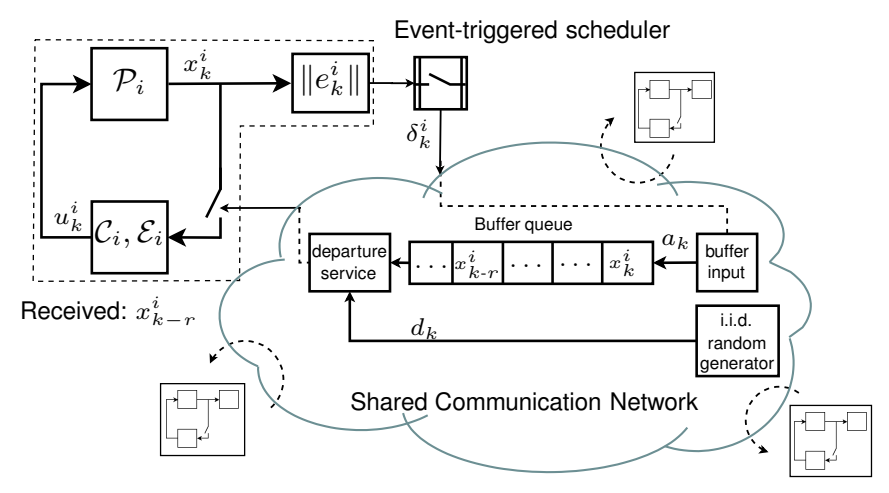

Fig. 1: Schematic of a multiple-loop NCS with a shared communication network equipped with state-dependent data queuing mechanism.

at time-step $k$ are represented by $a_{k}$ and $d_{k}$, respectively. Hence, the buffer dynamics can be expressed as

$$
l_{k+1}=l_{k}+a_{k}-d_{k}, \quad l_{k} \in \mathbb{N} \cup\{0\}, \forall k>0 .
$$

At every time-step, the controller $\mathcal{C}_{i}$ either receives new state information (delayed or un-delayed) or receives nothing. Let us generally assume that at a time-step $k$, the newest state information the controller $\mathcal{C}_{i}$ has access to, is $x_{\bar{k}}^{i}, \bar{k} \leq k$, which is received at one of the time-steps over $[\bar{k}, k]$. This means that $x_{\bar{k}}^{i}$ had been sent for transmission at time $\bar{k}$, and no newer data packet has been received by $\mathcal{C}_{i}$ afterwards. Therefore, the delay at time $k$ would be $k-\bar{k}$. Depending on the queue length $l_{\bar{k}}$ at time-step $\bar{k}$ and the buffer output, this induced delay, denoted by $\bar{\tau}_{k}^{i}$, is one of the integer values in the set $[0, k]$. It should be noted that, depending on how arrival and departure of the buffer are modeled, the induced delay $\bar{\tau}_{k}^{i}$ can be the outcome of a stochastic or deterministic process. We will discuss this comprehensively in the sequel.

Let the estimator installed at a controller side $\mathcal{C}_{i}$ has the local knowledge of its corresponding sub-system parameters $A_{i}, B_{i}, W_{i}$ and the distribution of $x_{0}^{i}$, together with the history of the control inputs $U_{[0, k-1]}^{i}=\left\{u_{0}^{i}, u_{1}^{i}, \ldots, u_{k-1}^{i}\right\}$, at time-step $k$. The state estimate $\hat{x}_{k \mid k}^{i}$ can then be computed, assuming that the latest received information is $x_{\bar{k}}^{i}$, as follows

$$
\begin{aligned}
\hat{x}_{k \mid k}^{i} & =\mathrm{E}\left[x_{k}^{i} \mid x_{k-\bar{\tau}_{k}^{i}}^{i}, U_{[0, k-1]}^{i}\right] \\
& =A_{i}^{\bar{\tau}_{k}^{i}} x_{\bar{k}}^{i}+A_{i}^{\bar{\tau}_{k}^{i}-1} B_{i} u_{\bar{k}}^{i}+\ldots+A_{i} B_{i} u_{k-2}^{i}+B_{i} u_{k-1}^{i} .
\end{aligned}
$$

Having (3), the $i^{\text {th }}$ system posteriori and apriori estimation errors at time-step $k$, denoted by $e_{k \mid k}^{i}$ and $e_{k \mid k-1}^{i}$, evolve as

$$
\begin{gathered}
e_{k \mid k}^{i} \triangleq x_{k}^{i}-\mathrm{E}\left[x_{k}^{i} \mid x_{k-\bar{\tau}_{k}^{i}}^{i}, \bar{\tau}_{k}^{i}>0\right]=\sum_{r=1}^{\bar{\tau}_{k}^{i}} A_{i}^{r-1} w_{k-r}^{i}, \\
e_{k \mid k-1}^{i} \triangleq x_{k}^{i}-\mathrm{E}\left[x_{k}^{i} \mid x_{k-1-\bar{\tau}_{k-1}^{i}}^{i}\right]=\sum_{r=1}^{1+\bar{\tau}_{k-1}^{i}} A_{i}^{r-1} w_{k-r}^{i} .
\end{gathered}
$$

It is clear that for $\bar{\tau}_{k}^{i}=0$, we have $\hat{x}_{k \mid k}^{i}=x_{k}^{i}$, and consequently, $e_{k \mid k}^{i}=0$. From (5), if $\bar{\tau}_{k-1}^{i}=0$, then $e_{k \mid k-1}^{i}=w_{k-1}^{i}$. Let the state feedback control input $u_{k}^{i}$ for each sub-system $i$ be computed according to the following causal mapping of the $i^{\text {th }}$ sub-system's past information, i.e.

$$
u_{k}^{i}=-K_{k}^{i} \hat{x}_{k \mid k}^{i},
$$


where, $K_{k}^{i}$ is any control gain designed for the ideal communication case without resource limitations such that the closed-loop matrix $\left(A_{i}-B_{i} K_{k}^{i}\right)$ is Hurwitz. From (1), (4), and (6), the closed-loop dynamics for each sub-system $i \in$ $\{1, \ldots, N\}$ is

$$
\begin{aligned}
x_{k+1}^{i} & =\left(A_{i}-B_{i} K_{k}^{i}\right) x_{k}^{i}+B_{i} K_{k}^{i} e_{k \mid k}^{i}+w_{k}^{i} \\
& =\left(A_{i}-B_{i} K_{k}^{i}\right) x_{k}^{i}+B_{i} K_{k}^{i} \sum_{r=1}^{\bar{\tau}_{k}^{i}} A_{i}^{r-1} w_{k-r}^{i}+w_{k}^{i} .
\end{aligned}
$$

From (7) it follows that dynamics of each sub-system $i$ depend not only on its local I/O variables, but also on the queuing delays $\bar{\tau}_{k}^{i}$,s. Here is where the couplings between sub-systems appear as the result of sharing the communication network. Therefore, to analyze the behavior of local sub-systems and also of the overall networked system, timevarying queuing delays that act as the cross-layer coupling variable between sub-systems, need to be taken into account.

Remark 1: In control systems where delay is unknown, control actions are computed based on the latest received updates, i.e. $u_{t}^{i}=-K_{t}^{i} x_{t-\tau_{t}^{i}}$. The control gain $K_{t}^{i}$ then plays a role in characterizing stability, i.e. not any control gain which stabilizes the un-delayed system can be used for the delayed system as well. Here we assume that each control loop knows the delay of the information it receives, and therefore, the control policy uses state estimation. Hence, under the assumed information structure, control actions are computed according to the certainty equivalence control (6). This ensures the induced delay appears only in the estimation error, which is unaffected by the control gain (see (5)).

\section{A. State-independent sampling with FIFO buffer service}

Let us assume that both buffer input and output are stateindependent. In the sequel, we first study deterministic and then stochastic input and output scenarios.

1) Deterministic buffer input and output: As a general static deterministic scenario, we assume a constant buffer input $\bar{n} \leq N$ at each time-step. Buffer output is also static based on the FIFO model, i.e. packets at the front of the queue are released when bandwidth is assigned. Note that, often in the existing literature, the buffer queue is elongated based on "first in" model, i.e. data can arrive at the buffer at any time instance. In discrete time NCSs where the sampling durations of control systems are identical, however, the transmission requests may arrive at the buffer simultaneously. Without loss of any generality, throughout this paper we assume that transmission requests at one time-step are randomly located at the queue tail, via a biased or unbiased randomization. Hence, the maximum delay for a sub-system corresponds to the case that its data packet is queued as the last one among all packets sent to the buffer. Assuming that $x_{\bar{k}}^{i}$ is queued at time $\bar{k}$ with the then buffer length $l_{\bar{k}}$, together with having $a_{k}=\bar{n}$, and buffer output $d_{k}=d_{c}$ for all $k$, we have

$$
\max \left\{\bar{\tau}_{k}^{i}\right\}=\left\lfloor\frac{l_{\bar{k}}+\bar{n}}{d_{c}}\right\rfloor,
$$

where, $\lfloor\cdot\rfloor$ is the floor operator. It is clear from (8) that if $d_{c} \geq \bar{n}$, then $\max \left\{\bar{\tau}_{k}^{i}\right\} \leq \frac{l_{0}}{d_{c}}$. Otherwise, if $d_{c}<\bar{n}$, then $l_{k} \rightarrow \infty$ as $k \rightarrow \infty$, and asymptotically $\max \left\{\bar{\tau}_{k}^{i}\right\} \rightarrow \infty$.
2) Stochastic buffer input and output: Consider that $a_{k}$ and $d_{k}$ are i.i.d. positive integer-valued random variables chosen at each time from finite-moment discrete distributions with respective means $\mu_{a}$ and $\mu_{d}$. Assume $x_{\bar{k}}^{i}$ is queued at time $\bar{k}$ as the latest data packet among all the transmission requests at time $\bar{k}$. From (2), the queue length at time $\bar{k}$ is

$$
\begin{aligned}
l_{\bar{k}} & =l_{0}+\left(a_{0}+a_{1}+\ldots+a_{\bar{k}-1}\right)-\left(d_{0}+d_{1}+\ldots+d_{\bar{k}-1}\right) \\
& =l_{0}+\sum_{r=0}^{\bar{k}-1}\left(a_{r}-d_{r}\right) .
\end{aligned}
$$

If $x_{\bar{k}}^{i}$ is received by $\mathcal{C}_{i}$ at an arbitrary time $k$, then $x_{\bar{k}}^{i}$ should have been discharged from the buffer at time-step $k$, while at time $k-1, x_{\bar{k}}^{i}$ should have still stayed in the buffer. Mathematically, the following two inequalities should hold:

$$
\begin{aligned}
& a_{\bar{k}}+l_{\bar{k}}-\left(d_{\bar{k}}+d_{\bar{k}+1}+\ldots+d_{k-1}\right)>0, \\
& a_{\bar{k}}+l_{\bar{k}}-\left(d_{\bar{k}}+d_{\bar{k}+1}+\ldots+d_{k-1}+d_{k}\right) \leq 0 .
\end{aligned}
$$

Substituting $l_{\bar{k}}$ from expression (9) and taking the expectation from (10), for an arbitrary receiving time $k$, we obtain

$$
\begin{aligned}
& \mathrm{E}\left[a_{\bar{k}}+l_{0}+\sum_{r=0}^{\bar{k}-1}\left(a_{r}-d_{r}\right)-\left(d_{\bar{k}}+d_{\bar{k}+1}+\ldots+d_{k-1}\right)\right] \\
& \quad=l_{0}+\mathrm{E}\left[\sum_{r=0}^{\bar{k}} a_{r}-\sum_{s=0}^{\bar{k}} d_{s}-\sum_{s=\bar{k}+1}^{k-1} d_{s}\right] \\
& \quad=l_{0}+(\bar{k}+1)\left(\mu_{a}-\mu_{d}\right)-\mathrm{E}[(k-\bar{k}-1)] \mu_{d} .
\end{aligned}
$$

With similar calculations this time with the inequality (11), the following bounds for the delay can be derived

$$
\frac{l_{0}+(\bar{k}+1)\left(\mu_{a}-\mu_{d}\right)}{\mu_{d}} \leq \mathrm{E}\left[\bar{\tau}_{k}^{i}\right]<\frac{l_{0}+(\bar{k}+1)\left(\mu_{a}-\mu_{d}\right)}{\mu_{d}}+1
$$

It is clear that for $\mu_{d} \geq \mu_{a}$, we have $\frac{l_{0}}{\mu_{d}} \leq \mathrm{E}\left[\bar{\tau}_{k}^{i}\right]<\frac{l_{0}}{\mu_{d}}+1$.

Remark 2: From (8) and (13) it follows that for stateindependent data queuing, either deterministic or stochastic, sufficient queue stability in terms of mean-square boundedness of the queue length is guaranteed only if the steady state buffer input and buffer output are equally balanced, which results in the delay being asymptotically bounded. Otherwise, delay converges to infinity and so does the waiting queue.

\section{EVENT-BASED SCHEDULING \& STATE-DEPENDENT BUFFER SERVICE}

In this section, let each sub-system $i$ be equipped with an event-triggered scheduler $\mathcal{S}_{i}$ which decides at each time $k>0$ whether to send new data to the buffer or not. The buffer output $d_{k}$ is assumed to be randomly selected from i.i.d. discrete distributions with mean $\mu_{d}>0$. We denote the $i^{\text {th }}$ scheduler's binary-valued decision at time $k$ by $\delta_{k}^{i}$, and

$$
\delta_{k}^{i}= \begin{cases}1, & x_{k}^{i} \text { sent to the buffer for transmission } \\ 0, & x_{k}^{i} \text { not sent to the buffer }\end{cases}
$$

According to (14), the buffer input $a_{k}$ can be expressed as

$$
a_{k}=\sum_{j=1}^{N} \delta_{k}^{j}
$$

The information available at the scheduler $\mathcal{S}_{i}$ to decide on $\delta_{k}^{i}$ is $\left\{U_{[0, k-1]}^{i}, x_{k}^{i}, x_{k-1-\bar{\tau}_{k-1}^{i}}^{i}, \bar{\tau}_{k-1}^{i}\right\}$. The decision variable 
$\delta_{k}^{i}$ is then generated at every time-step $k$ as the outcome of the following local event-triggered threshold-based policy

$$
\delta_{k}^{i}= \begin{cases}1, & \text { if } \quad\left\|e_{k \mid k-1}^{i}\right\|_{2}^{2}>\eta_{i} \\ 0, & \text { if } \quad\left\|e_{k \mid k-1}^{i}\right\|_{2}^{2} \leq \eta_{i}\end{cases}
$$

where, the constant $\eta_{i} \geq 0$ is the error threshold for subsystem $i$. From (16) it follows that $\delta_{k}^{i}$ 's are functions of $\left\|e_{k \mid k-1}^{i}\right\|_{2}^{2}$. This means $a_{k}$ in (15) is dynamically coupled with all sub-systems error states. In addition, from (5), $e_{k \mid k-1}^{i}$ depends on $A_{i}, \bar{\tau}_{k-1}^{i}$, and $w_{k-r}^{i}$, with $r \in\left[1,1+\bar{\tau}_{k-1}^{i}\right]$. Thus, $e_{k \mid k-1}^{i}$ and also $\left\|e_{k \mid k-1}^{i}\right\|_{2}^{2}$ are both random variables. Consequently, $a_{k}$ is random and dependent on statistical properties of $\left\|e_{k \mid k-1}^{i}\right\|_{2}^{2}$, for all $i \in\{1, \ldots, N\}$ with $\delta_{k}^{i}=1$.

For simplifying the derivations, we assume that the system matrices $A_{i}$ 's are diagonal. This assumption does not result in loss of any generality, but guarantees that $e_{k \mid k-1}^{i}$ is a random vector with independent normally distributed random elements ${ }^{1}$. Hence, $e_{k \mid k-1}^{i}$ is a multi-variate normally distributed random vector with delay-dependent covariance. Finally, $\left\|e_{k \mid k-1}^{i}\right\|_{2}^{2}$ 's distribution becomes sum of independent gamma distributions, with the following parameters

$$
\left\|e_{k \mid k-1}^{i}\right\|_{2}^{2} \sim \sum_{l=1}^{n_{i}} \Gamma\left(\frac{1}{2}, 2 \sigma_{j j}^{2}\right)
$$

where, $\sigma_{j j}$ is the element on the $j^{\text {th }}$ row and column of the diagonal covariance matrix $\Sigma_{e}=\sum_{r=1}^{1+\bar{\tau}_{k-1}^{i}} A_{i}^{r-1} W_{i} A_{i}^{r-1^{\top}}$, and $n_{i}$ is the dimension of $e_{k \mid k-1}^{i}$. We define a new vectorvalued random sequence $E$, evolved until time $k$, as follows

$$
E \triangleq\left\{\mathcal{E}_{0}, \mathcal{E}_{1}, \ldots, \mathcal{E}_{k}\right\}
$$

where, $\mathcal{E}_{t}=\left[\left\|e_{t \mid t-1}^{1}\right\|_{2}^{2}, \ldots,\left\|e_{t \mid t-1}^{N}\right\|_{2}^{2}\right]^{\top}$ is a random vector containing independent random elements each distributed according to (17). From (15)-(17), the expected buffer input, assuming that $\bar{\tau}_{k-1}^{i}$ is known for sub-system $i$, becomes

$$
\begin{aligned}
& \mathrm{E}\left[a_{k} \mid \bar{\tau}_{k-1}^{j}, \eta_{j}, \forall j\right]=\sum_{j=1}^{N} \mathrm{E}\left[\delta_{k}^{j}\right]=\sum_{j=1}^{N} \mathrm{P}\left[\delta_{k}^{j}=1\right] \\
& =\sum_{j=1}^{N} \mathrm{P}\left[\left\|e_{k \mid k-1}^{j}\right\|_{2}^{2}>\eta_{j}\right]=\sum_{j=1}^{N}\left(1-F_{\mathcal{E}_{k}^{j}}\left(\eta_{j}\right)\right),
\end{aligned}
$$

where, $F_{\mathcal{E}_{k}^{j}}\left(\eta_{j}\right)$ is the value of the cumulative distribution function $(\mathrm{CDF})$ of the $j^{\text {th }}$ element of $\mathcal{E}_{k}$, computed at $\eta_{j}$.

As the buffer outputs $d_{k}$ are i.i.d. random variables, and $a_{k}$ is also random at every time-step $k$, hence $\bar{\tau}_{k-1}^{i}$ is a discrete random variable. Therefore, to compute the expectation of $a_{k}$ for any delay variable $\bar{\tau}_{k-1}^{i}$, we need to derive the marginal distribution of $\left\|e_{k \mid k-1}^{j}\right\|_{2}^{2}$, averaged over all $\bar{\tau}_{k-1}^{i}$ 's. Let us define a discrete-vector-valued random sequence $\tau$, as

$$
\tau \triangleq\left\{\tau_{0}, \tau_{1}, \ldots, \tau_{k}\right\}
$$

where, $\tau_{t}=\left[\tau_{t}^{1}, \ldots, \tau_{t}^{N}\right]^{\top}$, and each discrete random variable $\tau_{t}^{i}, i \in\{1, \ldots, N\}$ takes its value from the discrete set $[0, t]$. As before, we denote the realization of $\tau_{t}^{i}$ by $\bar{\tau}_{t}^{i}$. Since

\footnotetext{
${ }^{1}$ Relaxing this assumption means $e_{k \mid k-1}^{i}$ has statistically dependent normally distributed elements, and makes the mathematics more complicated. The results of this paper, however, extend to non-diagonal system matrices.
}

$\mathcal{E}_{k}^{i}$ is a continuous random variable at every time-step $k$, while $\tau_{k-1}^{i}$ is discrete, the joint density function, denoted by $f_{\mathcal{E}_{k}, \tau_{k-1}}^{i}$, should be expressed in mixed form, as follows:

$$
\begin{aligned}
& f_{\mathcal{E}_{k}, \tau_{k-1}}^{i}\left(\left\|e_{k \mid k-1}^{i}\right\|_{2}^{2}, \bar{\tau}_{k-1}^{i}\right)= \\
& f_{\mathcal{E}_{k} \mid \tau_{k-1}}^{i}\left(\left\|e_{k \mid k-1}^{i}\right\|_{2}^{2} \mid \bar{\tau}_{k-1}^{i}\right) \mathrm{P}\left(\tau_{k-1}^{i}=\bar{\tau}_{k-1}^{i}\right) .
\end{aligned}
$$

Accordingly, for the marginal distribution $f_{\mathcal{E}_{k}}^{i}\left(\left\|e_{k \mid k-1}^{i}\right\|_{2}^{2}\right)$ averaged over all delay variables $\tau_{k-1}^{i}$, we can write

$f_{\mathcal{E}_{k}}^{i}\left(\left\|e_{k \mid k-1}^{i}\right\|_{2}^{2}\right)=\sum_{\bar{\tau}_{k-1}^{i}} f_{\mathcal{E}_{k}, \tau_{k-1}}^{i}\left(\mathcal{E}_{k}\left\|e_{k \mid k-1}^{i}\right\|_{2}^{2}, \bar{\tau}_{k-1}^{i}\right)$.

Expression (21) requires knowing $\mathrm{P}\left(\tau_{k-1}^{i}=\bar{\tau}_{k-1}^{i}\right)$, which is correlated with the buffer input and output. To take this into account, let $\bar{\tau}_{k-1}^{i}$ be an arbitrary realization of $\tau_{k-1}^{i}$, for the random time $k-1$. The time of being queued is then $\tilde{k}=k-1-\bar{\tau}_{k-1}^{i}$. Let $x_{\tilde{k}}^{i}$ be received by $\mathcal{C}_{i}$ at an arbitrary time-step $k-1$. Then the following two inequalities hold

$$
\begin{aligned}
& a_{\tilde{k}}+l_{\tilde{k}}>\left(d_{\tilde{k}}+d_{\tilde{k}+1}+\ldots+d_{k-2}\right), \\
& a_{\tilde{k}}+l_{\tilde{k}} \leq\left(d_{\tilde{k}}+d_{\tilde{k}+1}+\ldots+d_{k-1}\right) .
\end{aligned}
$$

Since the left sides of the above inequalities are not random, and also, as the outputs $d_{k}$ are i.i.d. at all $k$, we arrive to

$$
\mathrm{P}\left[\tau_{k-1}^{i}=\bar{\tau}_{k-1}^{i}\right]=\mathrm{P}\left[a_{\tilde{k}}+l_{\tilde{k}} \leq \sum_{t=\tilde{k}}^{k-1} d_{t}\right] \mathrm{P}\left[a_{\tilde{k}}+l_{\tilde{k}}>\sum_{t=\tilde{k}}^{k-2} d_{t}\right]
$$

From (15), we know $a_{\tilde{k}}=\sum_{j=1}^{N} \delta_{\tilde{k}}^{j}$. From (2), we conclude

$$
a_{\tilde{k}}+l_{\tilde{k}}=l_{0}+\sum_{t=0}^{\tilde{k}} \sum_{j=1}^{N} \delta_{t}^{j}-\sum_{t=0}^{\tilde{k}-1} d_{t} .
$$

We can then compute the two following probabilities:

$$
\begin{aligned}
& \mathrm{P}\left[a_{\tilde{k}}+l_{\tilde{k}} \leq \sum_{t=\tilde{k}}^{k-1} d_{t}\right]=\mathrm{P}\left[l_{0}+\sum_{t=0}^{\tilde{k}} \sum_{j=1}^{N} \delta_{t}^{j}-\sum_{t=0}^{\tilde{k}-1} d_{t} \leq \sum_{t=\tilde{k}}^{k-1} d_{t}\right], \\
& \mathrm{P}\left[a_{\tilde{k}}+l_{\tilde{k}}>\sum_{t=\tilde{k}}^{k-2} d_{t}\right]=\mathrm{P}\left[l_{0}+\sum_{t=0}^{\tilde{k}} \sum_{j=1}^{N} \delta_{t}^{j}-\sum_{t=0}^{\tilde{k}-1} d_{t}>\sum_{t=\tilde{k}}^{k-2} d_{t}\right] .
\end{aligned}
$$

Note that all the variables above except the buffer outputs $\left\{d_{\tilde{k}}, \ldots, d_{k-2}, d_{k-1}\right\}$ are known and not random. In fact, the only random term in the first expression is $\sum_{t=\tilde{k}}^{k-1} d_{t}$, and in the second expression is $\sum_{t=\tilde{k}}^{k-2} d_{t}$. Assuming that the distribution of the i.i.d. buffer outputs $d_{k}$ is known for all $k>0$, the probability mass functions (PMFs) of the two discrete random variables $\sum_{t=\tilde{k}}^{k-1} d_{t}$, and $\sum_{t=\tilde{k}}^{k-2} d_{t}$, denoted respectively by $F_{d_{[\tilde{k}, k-1]}}^{i}$, and $F_{d_{[\tilde{k}, k-2]}^{i}}^{i}$, are expressed as

$$
\begin{aligned}
& \mathrm{P}\left[a_{\tilde{k}}+l_{\tilde{k}} \leq \sum_{t=\tilde{k}}^{k-1} d_{t}\right]=1-F_{d_{[\tilde{k}, k-1]}}^{i}\left(l_{0}+\sum_{t=0}^{\tilde{k}} \sum_{j=1}^{N} \delta_{t}^{j}-\sum_{t=0}^{\tilde{k}-1} d_{t}\right) \text {, } \\
& \mathrm{P}\left[a_{\tilde{k}}+l_{\tilde{k}}>\sum_{t=\tilde{k}}^{k-2} d_{t}\right]=F_{d_{[\tilde{k}, k-2]}}^{i}\left(l_{0}+\sum_{t=0}^{\tilde{k}} \sum_{j=1}^{N} \delta_{t}^{j}-\sum_{t=0}^{\tilde{k}-1} d_{t}\right) .
\end{aligned}
$$

Finally, $\mathrm{P}\left[\tau_{k-1}^{i}=\bar{\tau}_{k-1}^{i}\right]$ can be re-written as

$$
\mathrm{P}\left[\tau_{k-1}^{i}=\bar{\tau}_{k-1}^{i}\right]=F_{d_{[\tilde{k}, k-2]}}^{i}\left(a_{\tilde{k}}+l_{\tilde{k}}\right)\left(1-F_{d_{[\tilde{k}, k-1]}}^{i}\left(a_{\tilde{k}}+l_{\tilde{k}}\right)\right) .
$$


Having the latter expression, the marginal distribution expressed in (22), is then fully characterized, according to (21). Define the marginal CDF of $\left\|e_{k \mid k-1}^{i}\right\|_{2}^{2}$, associated with the marginal distribution function (22), as $F_{\mathcal{E}_{k}^{i}}^{\operatorname{margin}}\left(\left\|e_{k \mid k-1}^{i}\right\|_{2}^{2}\right)$. Then, the unconditional expected buffer arrival, i.e., $\mathrm{E}\left[a_{k}\right]$ at any time-step $k>0$, can be expressed as follows

$$
\mathrm{E}\left[a_{k}\right]=\sum_{j=1}^{N} \mathrm{P}\left[\delta_{k}^{j}=1\right]=\sum_{j=1}^{N}\left(1-F_{\mathcal{E}_{k}^{j}}^{\operatorname{margin}}\left(\eta_{j}\right)\right) .
$$

It is concluded from (24) that $\mathrm{E}\left[a_{k}\right]$ at any general time $k$ is determined by the error thresholds $\eta_{j}$, for all $j$, and the statistical properties of the buffer outputs $\left\{d_{0}, d_{1}, \ldots, d_{k-1}\right\}$.

\section{Stability Analysis}

In this section, we study asymptotic stability of the described NCS under the proposed state-dependent data queuing, and derive almost sure sufficient mean-square stability condition. Let us first define the aggregate state vectors $e_{k \mid k-1} \triangleq\left[e_{k \mid k-1}^{1^{\top}}, \ldots, e_{k \mid k-1}^{N^{\top}}\right]^{\top}$, and $x_{k} \triangleq\left[x_{k}^{1^{\top}}, \ldots, x_{k}^{N^{\top}}\right]^{\top}$, at time $k$. Together with $l_{k} \in \mathbb{R}^{+} \cup\{0\}$, we can characterize the dynamics of the NCS at every time $k$ by the overall state vector $\left[x_{k}^{\top}, e_{k \mid k-1}^{\top}, l_{k}\right]^{\top}$. Note that, the overall system with the mentioned state vector is not linear, due to the general non-linear coupling between $e_{k \mid k-1}$, and $l_{k}$ via $\bar{\tau}_{k-1}^{i}$ (see (5)). Within each sub-system, however, the local aggregate state $\left[x_{k}^{i^{\top}}, e_{k \mid k-1}^{i^{\top}}\right]^{\top}$ is linear, according to (5) and (7). Recalling Remark 1 , and assuming controllability of each pair $\left(A_{i}, B_{i}\right)$, it is guaranteed that the overall NCS is meansquare stable in the absence of network-induced delay. This can also be concluded from (7), as for $\bar{\tau}_{k}^{i}=0$, it reduces to $x_{k+1}^{i}=\left(A_{i}-B_{i} K_{k}^{i}\right) x_{k}^{i}+w_{k}^{i}$. In the presence of induced-delay, however, the system state is affected by the estimation error, which is itself independent of $x_{k}^{i}$. Thus, mean-square stability holds only if $e_{k \mid k-1}^{i}$ is mean-square stable, that is ensured only if delay is finite, according to (5). In addition, since $d_{k}>0$ for all $k$, and $l_{0}<\infty$, boundedness of delay is ensured if $l_{k}$ is bounded. In the following, we derive the sufficient NCS stability condition in almost sure mean-square sense. First, we revisit the following lemma about convergence of linear sequences.

Lemma 1: [22, Chapter 1] Consider a linear first-order non-homogeneous sequence $\left\{s_{k}\right\}$, as follows

$$
s_{k+1}=s_{k}+b, \quad k=0,1,2, \ldots, s_{0}=c .
$$

Then, the following statements hold:

1) if $b>0$, or $b<0$, the sequence diverges to $+\infty$, and $-\infty$, respectively,

2) if $b=0, s_{k}$ is a constant sequence.

Theorem 1: Consider an NCS with $N$ dynamical subsystems described in (1), each with estimation and control processes given in (3) and (6), respectively. Assume that the shared network is equipped with a FIFO buffer with initial queue length $l_{0}$, and output $d_{k}$, where $d_{k}$ is an i.i.d. discrete random variable with bounded mean $\mu_{d}>0$ at each time $k$. Under the event-triggered law (16), there always exist local thresholds $\eta_{i}$ 's, $i \in\{1, \ldots, N\}$, such that for any $\mu_{d}>0$,

$$
\mathrm{E}\left[l_{k}\right]=l_{0}, \quad \forall k=0,1,2, \ldots
$$

Proof: From (2) and (15), the queue dynamics become

$$
l_{k+1}=l_{k}+\sum_{i=1}^{N} \delta_{k}^{i}-d_{k} .
$$

Taking expectation from both sides of (25) yields

$$
\mathrm{E}\left[l_{k+1}\right]=\mathrm{E}\left[l_{k}\right]+\mathrm{E}\left[\sum_{i=1}^{N} \delta_{k}^{i}-d_{k}\right]
$$

It follows from Lemma 1 that the length sequence $\left\{\mathrm{E}\left[l_{k}\right]\right\}$ is a non-divergent time sequence, only if at any time-step $k$, $\mathrm{E}\left[\sum_{i=1}^{N} \delta_{k}^{i}-d_{k}\right]=0$. Since negative length is meaningless and is projected to zero, we can replace the equality with inequality $\mathrm{E}\left[\sum_{i=1}^{N} \delta_{k}^{i}-d_{k}\right] \leq 0$. Employing (24), we have

$$
\mathrm{E}\left[\sum_{i=1}^{N} \delta_{k}^{i}-d_{k}\right]=\sum_{i=1}^{N}\left(1-F_{\mathcal{E}_{k}^{i}}^{\operatorname{margin}}\left(\eta_{i}\right)\right)-\mu_{d} .
$$

Hence $\mathrm{E}\left[l_{k}\right]$ is a constant sequence, and $\mathrm{E}\left[l_{k}\right]=l_{0}$, if

$$
\sum_{i=1}^{N}\left(1-F_{\mathcal{E}_{k}^{i}}^{\text {margin }}\left(\eta_{i}\right)\right) \leq \mu_{d}
$$

for all $k>0$. We can then find a set of feasible thresholds $\eta_{i} \geq 0, i \in\{1, \ldots, N\}$ such that for any $0<\mu_{d}<N$, (26) holds. It is clear that for all $\mu_{d} \geq N$, the inequality (26) is satisfied for any $\eta_{i}$, and the proof then readily follows.

It follows from Theorem 1 that the smaller the mean of the buffer output is, the larger the error thresholds $\eta_{i}$ 's should become to hold the queue stability, which clearly results in a lengthy time gap between two consecutive transmissions.

Corollary 1: Consider the inequality (26). Then, the nonnegative random variable $\tau_{k}^{i}$ is integrable, and any realization of $\tau_{k}^{i}$, i.e. $\bar{\tau}_{k}^{i} \in\{0,1, \ldots, k\}$, satisfies $\bar{\tau}_{k}^{i}<\infty$, almost surely, or equivalently, $\mathrm{P}\left[\bar{\tau}_{k}^{i}<\infty\right]=1$, for all $k \in\{1,2, \ldots\}$.

Proof: With similar calculations in deriving the expression (13), we can find the following bounds for the expected delay $\mathrm{E}\left[\tau_{k}^{i}\right]=\mathrm{E}[k]-\bar{k}$, where $k$ is the expected arrival time for a data packet being queued at an arbitrary time-step $\bar{k}$

$$
\frac{\mathrm{E}\left[a_{\bar{k}}\right]+\mathrm{E}\left[l_{\bar{k}}\right]}{\mu_{d}} \leq \mathrm{E}\left[\tau_{k}^{i}\right]<\frac{\mathrm{E}\left[a_{\bar{k}}\right]+\mathrm{E}\left[l_{\bar{k}}\right]}{\mu_{d}}+1 .
$$

From the expressions (2) and (24), we have

$$
\frac{\mathrm{E}\left[a_{\bar{k}}\right]+\mathrm{E}\left[l_{\bar{k}}\right]}{\mu_{d}}=\frac{\sum_{t=1}^{\bar{k}} \sum_{j=1}^{N}\left(1-F_{\mathcal{E}_{t}^{j}}^{\operatorname{margin}}\left(\eta_{j}\right)\right)-\bar{k} \mu_{d}+l_{0}}{\mu_{d}} .
$$

Having (26) satisfied at every time-step $k>0$, we obtain

$$
\frac{l_{0}}{\mu_{d}} \leq \mathrm{E}\left[\tau_{k}^{i}\right]<\frac{l_{0}}{\mu_{d}}+1
$$

Boundedness of $\mathrm{E}\left[\tau_{k}^{i}\right]$ confirms integrability of $\tau_{k}^{i}$, and it is then straightforward to show, by the definition of Lebesgue integral, that any realization of the random variable $\tau_{k}^{i}$ is almost surely finite, i.e. $\mathrm{P}\left[\bar{\tau}_{k}^{i}<\infty\right]=1$, for all $k>0$.

Theorem 2: Consider the NCS described in Theorem 1. Assuming that each pair $\left(A_{i}, B_{i}\right)$ is controllable, then a set of finite local error thresholds $\eta=\left\{\eta_{1}, \ldots, \eta_{N}\right\}$ exists such that, under the event-based scheduler (16), the NCS with the overall state $\left[x_{k}^{\top}, e_{k \mid k-1}^{\top}, l_{k}\right]^{\top}$ is a.s. mean-square stable. 
Proof: As already discussed, evolution of the networkinduced error $e_{k \mid k-1}^{i}$ is independent of $x_{k}^{i}$, hence asymptotic convergence of $e_{k \mid k-1}^{i}$ in mean square sense guarantees mean-square convergence of the local system states $x_{k}^{i}$,s, according to (7). To show this, we have from expression (5)

$$
\begin{aligned}
\mathrm{E}\left[\left\|e_{k \mid k-1}^{i}\right\|_{2}^{2}\right] & =\mathrm{E}\left[\left\|\sum_{r=1}^{1+\bar{\tau}_{k-1}^{i}} A_{i}^{r-1} w_{k-r}^{i}\right\|_{2}^{2}\right] \\
& =\mathrm{E}\left[\sum_{r=1}^{1+\bar{\tau}_{k-1}^{i}}\left\|A_{i}^{r-1} w_{k-r}^{i}\right\|_{2}^{2}\right],
\end{aligned}
$$

where, (28) holds due to statistical independence of noise realizations. Finding the exact expression for (28) is challenging since the stopping time, i.e. $\bar{\tau}_{k-1}^{i}$, determines the covariance of random elements in the summation via the term $A_{i}^{r-1}$. Thus we have a random summation of independent but not identically distributed random elements. We, therefore, consider the worst case scenario and find an upper-bound for the expectation of the $\mathcal{L}_{2}$ norm of the error. Assume that all sub-systems $i \in\{1, \ldots, N\}$ are unstable. Then, we have

$$
\begin{aligned}
& \mathrm{E}\left[\sum_{r=1}^{1+\bar{\tau}_{k-1}^{i}}\left\|A_{i}^{r-1} w_{k-r}^{i}\right\|_{2}^{2}\right] \leq \mathrm{E}\left[\sum_{r=1}^{1+\bar{\tau}_{k-1}^{i}}\left\|A_{i}^{\bar{\tau}_{k-1}^{i}} w_{k-r}^{i}\right\|_{2}^{2}\right] \\
& \quad \leq \mathrm{E}\left[\left\|A_{i}\right\|_{2}^{2 \bar{\tau}_{k-1}^{i}} \sum_{r=1}^{1+\bar{\tau}_{k-1}^{i}}\left\|w_{k-r}^{i}\right\|_{2}^{2}\right],
\end{aligned}
$$

where, the second inequality is ensured via sub-multiplicative property of matrix norms. From Corollary 1, we know that any delay realization $\bar{\tau}_{k-1}^{i}$ is a.s. bounded, i.e. a positive constant $M$ exists a.s., such that $\bar{\tau}_{k-1}^{i}<M<\infty$. Therefore,

$$
\mathrm{E}\left[\sum_{r=1}^{1+\bar{\tau}_{k-1}^{i}}\left\|A_{i}^{r-1} w_{k-r}^{i}\right\|_{2}^{2}\right] \leq\left\|A_{i}\right\|_{2}^{2 M} \mathrm{E}\left[\sum_{r=1}^{1+\bar{\tau}_{k-1}^{i}}\left\|w_{k-r}^{i}\right\|_{2}^{2}\right] .
$$

Since, $\left\|w_{k-r}^{i}\right\|_{2}^{2}$,s are i.i.d. for each $r \in\left[1,1+\bar{\tau}_{k-1}^{i}\right]$ and for all $i \in\{1, \ldots, N\}$, and additionally as $1+\bar{\tau}_{k-1}^{i}$ is now a stopping time ${ }^{2}$, we employ Wald's identity, and arrive to

$$
\mathrm{E}\left[\sum_{r=1}^{1+\bar{\tau}_{k-1}^{i}}\left\|w_{k-r}^{i}\right\|_{2}^{2}\right]=\mathrm{E}\left[\bar{\tau}_{k-1}^{i}\right] \mathrm{E}\left[\left\|w_{k-r}^{i}\right\|_{2}^{2}\right] .
$$

Finally, according to (27), we conclude

$$
\mathrm{E}\left[\left\|e_{k \mid k-1}^{i}\right\|_{2}^{2}\right] \leq\left(\frac{l_{0}}{\mu_{d}}+1\right)\left\|A_{i}\right\|_{2}^{2 M} \operatorname{tr}\left(W_{i}\right)
$$

which proves $\mathrm{E}\left[\left\|e_{k \mid k-1}^{i}\right\|_{2}^{2}\right]$ for each sub-system $i$ is a.s. bounded. In addition, the buffer state $l_{k}$ is the sole coupling point between the sub-systems and determines their delay periods. Theorem 1 ensures a set of thresholds $\left\{\eta_{1}, \ldots, \eta_{N}\right\}$ exists such that $l_{k}$ is mean-square bounded. Since $l_{k}$ is generic for all sub-systems, under the given thresholds, local error states $e_{k}^{i}$ 's are mean-square bounded, and this ensures mean-square boundedness of the local system states $x_{k}^{i}$,s, if stabilizing gains $K_{k}^{i}$,s exist. and the proof is complete.

Remark 3: According to (29), the upper-bound of the error variance depends on $W_{i}, A_{i}, \mu_{d}$, and $l_{0}$. As expected,

\footnotetext{
${ }^{2}$ A random time $N$ is said to be stopping time with respect to a stochastic process $\left\{X_{n}, n \geq 0\right\}$, if for each $n \geq 0$, the event $N=n$ is fully determined by the total information contained in $\left\{X_{0}, \ldots, X_{n}\right\}$, and $N$ is independent of the future states $\left\{X_{n+1}, X_{n+2}, \ldots\right\}$.
}

higher noise uncertainty, system matrices with larger spectral radius, or lower buffer output lead to increase the bound of the error variance. Therefore, although results of Theorem 2 hold for general parameters, if a specific performance is required, these parameters need to be appropriately adjusted.

\section{NUMERICAL RESULTS}

We consider multi-loop NCSs consisting of $N$ stochastic scalar sub-systems, where $N \in\{2,4,6,8,10\}$. Sub-systems are divided into two heterogeneous classes of $\frac{N}{2}$ identical systems, i.e. class of unstable and stable sub-systems, denoted by $c l_{1}$ and $c l_{2}$, respectively, with the parameters $A_{c l_{1}}=1.25, A_{c l_{2}}=0.75, B_{c l_{1}}=B_{c l_{2}}=1, W_{c l_{1}}=W_{c l_{2}}=1$.

Every sub-system is assumed to be controlled by a deadbeat control law $K_{k}^{i}=A_{i} B_{i}^{-1}$, at every time-step $k>0$. Data are sent to a single buffer with $l_{0}=0$. We perform Monte Carlo simulations, and plot the averages over 10 runs, with the time horizon of each simulation run set to be $T=5000$.

To check our simulation accuracy, we first set $\eta_{i}=0$ for all $i$ that results in $a_{k}=N$ at all $k$. We observe that for an NCS with $N$ systems the average error variance grows unbounded if $d_{k}<N$. As expected, $d_{k} \geq N$ results in undelayed transmissions at all time-steps, and the average error variance equals to the average noise variance, (see (5)). In the stochastic case also for $\mathrm{E}\left[d_{k}\right]<N$, error variances take very large values, while for $\mathrm{E}\left[d_{k}\right]=N$, it varies from run to run between bounded and unbounded values. The reason is that in simulations with finite samples, the numerical mean of $d_{k}$ is not exactly equal to $N$ but close to $N$.

In Table I, state-independent stochastic queuing is compared with the event-triggered data queuing. In the upper part of Table I the error variances for different NCS setups with their respective $\mathrm{E}\left[d_{k}\right]$ for the static-input stochastic-output case are given. Discrete buffer outputs $d_{k}$ are uniformly distributed at each time-step, i.e. $d_{k} \sim \mathcal{U}(1,2 N)$, and $\mathrm{E}\left[d_{k}\right]=$ $\frac{2 N+1}{2}$. As all $N$ sub-systems regularly send data to the buffer, therefore, the average transmission rate equals exactly one

\begin{tabular}{|c|c|c|c|c|c|}
\hline Number of plants $(\mathrm{N})$ & 2 & 4 & 6 & 8 & 10 \\
\hline \multicolumn{6}{|c|}{ Static stochastic data queuing } \\
\hline Error threshold $\left(\eta_{i}\right)$ & 0 & 0 & 0 & 0 & 0 \\
\hline $\mathrm{E}\left[d_{k}\right]$ & 2.5 & 4.5 & 6.5 & 8.5 & 10.5 \\
\hline Average error variance & 1.359 & 2.588 & 4.807 & 8.445 & 12.676 \\
\hline$\frac{\text { Total number of transmissions }}{N . T}$ & 1 & 1 & 1 & 1 & $\underline{1}$ \\
\hline \multicolumn{6}{|c|}{ Event-based stochastic data queuing } \\
\hline Error threshold $\left(\eta_{i}\right)$ & 0.25 & 7.00 & 19.80 & 46.15 & 78.20 \\
\hline $\mathrm{E}\left[d_{k}\right]$ & 2 & 4 & 6 & 8 & 10 \\
\hline Average error variance & 1.358 & 2.585 & 4.808 & 8.448 & $\underline{12.472}$ \\
\hline$\frac{\text { Total number of transmissions }}{N . T}$ & 0.649 & 0.102 & 0.059 & 0.047 & $\underline{0.042}$ \\
\hline$\mu_{d}^{\max }$ & 1.799 & 2.491 & 3.149 & 3.990 & 4.941 \\
\hline$\mu_{d}^{\text {average }}$ & 1.438 & 1.698 & 2.008 & 2.450 & 3.014 \\
\hline
\end{tabular}
transmission per time-step, per sub-system. Note that, as opposed to the deterministic case that if $d_{k} \geq N$, then average error variance reduces to the noise variance (in this case 1 ), in the stochastic case, the average error variance takes larger value. The reason is, in the latter case $d_{k}$ varies and

TABLE I: Static vs. event-based data queuing for shared resource NCSs 
TABLE II: Existence of stabilizing error thresholds for different $\mathrm{E}\left[d_{k}\right]<N$

\begin{tabular}{|c|c|c|c|}
\hline \multicolumn{4}{|c|}{$N=4$} \\
\hline $\mathrm{E}\left[d_{k}\right]$ & 1.5 & 2 & 2.5 \\
\hline Error threshold $\left(\eta_{i}\right)$ & 12.2 & 3.7 & 1.6 \\
\hline Average error variance & 3.516 & 1.996 & 1.776 \\
\hline$\mu_{d}^{\text {average }}$ & 1.488 & 1.922 & 2.208 \\
\hline \multicolumn{4}{|c|}{$N=6$} \\
\hline $\mathrm{E}\left[d_{k}\right]$ & 2.5 & 3 & 3.5 \\
\hline Error threshold $\left(\eta_{i}\right)$ & 8.2 & 3.5 & 2.1 \\
\hline Average error variance & 2.844 & 1.836 & 1.678 \\
\hline$\mu_{d}^{\text {average }}$ & 2.489 & 2.920 & 3.135 \\
\hline \multicolumn{4}{|c|}{$N=8$} \\
\hline $\mathrm{E}\left[d_{k}\right]$ & 3.5 & 4 & 4.5 \\
\hline Error threshold $\quad\left(\eta_{i}\right)$ & 6.4 & 3.3 & 2.5 \\
\hline Average error variance & 2.469 & 1.896 & 1.737 \\
\hline$\mu_{d}^{\text {average }}$ & 3.487 & 3.928 & 4.085 \\
\hline \multicolumn{4}{|c|}{$N=10$} \\
\hline $\mathrm{E}\left[d_{k}\right]$ & 4.5 & 5 & 5.5 \\
\hline Error threshold $\left(\eta_{i}\right)$ & 5.4 & 3.5 & 2.8 \\
\hline Average error variance & 2.341 & 1.946 & 1.801 \\
\hline$\mu_{d}^{\text {average }}$ & 4.476 & 4.849 & 4.976 \\
\hline
\end{tabular}

its realization is occasionally less than $N$, which results in delay, and consequently increases the estimation error.

In the lower part of Table I, we found error thresholds which provide similar error variances as those derived from the state-independent design for each setup. The results demonstrate that we can achieve very similar performance by considerably less transmissions. For example, for $N=10$ we achieve error variance 12.676 with $\mathrm{E}\left[d_{k}\right]=10.5$, with 50,000 transmissions for static data queuing. With event-based queuing similar performance is achieved by only 2,100 transmissions (see the underlined values). Moreover, from the sufficient stability condition (26), the maximum required $\mathrm{E}\left[d_{k}\right]$ to provide such performance is 4.941 , which compared to 10.5 for state-independent case is about $53 \%$ lower.

In Table II, we validate the results of Theorem 2 by showing the existence of $\eta_{i}$ 's for different $\mathrm{E}\left[d_{k}\right]<N$ that result in bounded error variances. The selected $\mathrm{E}\left[d_{k}\right]$ 's in Table II lead to overall instability for static data queuing. As expected, error thresholds must be larger for lower $\mathrm{E}\left[d_{k}\right]$. In Table II, $\mu_{d}^{a v e}$ 's are calculated instead of the maximum required $\mu_{d}^{\max }$ in order to observe an interesting property of the stochastic buffer discharge model. It is seen that $\mu_{d}^{a v e}$ is a reliable choice for $\mu_{d}$ if the standard deviation of the output distribution is small, and it becomes less reliable as the boundaries of the uniform distribution move afar.

\section{CONCLUSION}

In this paper, state-dependent data queuing in sharedresource multi-loop NCSs is addressed. We propose a crosslayer design framework within which local event-based schedulers decide if a data packet should be sent for transmission or not. Consequently, the buffer input becomes statedependent and the buffer state then depends on all control loop's estimation errors. A sufficient stability condition for the NCSs of interest is derived under the proposed statedependent scheme in mean-square sense. Moreover, perfor- mance improvement is shown to be achieved under our proposed design compared to the state-independent scenario.

\section{REFERENCES}

[1] R. A. Gupta and M. Y. Chow, "Networked control system: Overview and research trends," IEEE Transactions on Industrial Electronics, vol. 57, no. 7, pp. 2527-2535, 2010.

[2] J. Baillieul and P. J. Antsaklis, "Control and communication challenges in networked real-time systems," Proceedings of the IEEE, vol. 95, no. 1, pp. 9-28, 2007.

[3] M. H. Mamduhi, A. Molin, and S. Hirche, "On the stability of prioritized error-based scheduling for resource-constrained networked control systems*," IFAC Proceedings Volumes, vol. 46, no. 27, pp. 356 - 362, 2013. 4th IFAC Workshop on Distributed Estimation and Control in Networked Systems (2013).

[4] K. J. Åstrom and B. Bernhardsson, "Comparison of riemann and lebesgue sampling for first order stochastic systems," in Proc. of 41st IEEE Conf. on Decision and Control, vol. 2, pp. 2011-2016, 2002.

[5] X. Wang and M. D. Lemmon, "Event-triggering in distributed networked control systems," IEEE Transactions on Automatic Control, vol. 56, pp. 586-601, March 2011.

[6] D. V. Dimarogonas, E. Frazzoli, and K. H. Johansson, "Distributed event-triggered control for multi-agent systems," IEEE Transactions on Automatic Control, vol. 57, pp. 1291-1297, May 2012.

[7] D. Maity and J. S. Baras, "Event based control of stochastic linear systems," in 2015 International Conference on Event-based Control, Communication, and Signal Processing (EBCCSP), pp. 1-8, 2015.

[8] M. H. Mamduhi, A. Molin, D. Tolic, and S. Hirche, "Error-dependent data scheduling in resource-aware multi-loop networked control systems," Automatica, vol. 81, pp. 209 - 216, 2017.

[9] B. Sinopoli, L. Schenato, M. Franceschetti, K. Poolla, and S. Sastry, "An LQG optimal linear controller for control systems with packet losses," in Proc. of the 44th IEEE Conf. on Decision and Control, pp. $458-463,2005$

[10] B. A. Khashooei, D. J. Antunes, and W. P. M. H. Heemels, "Outputbased event-triggered control with performance guarantees," IEEE Trans. on Automatic Control, vol. 62, no. 7, pp. 3646-3652, 2017.

[11] P. Tabuada, "Event-triggered real-time scheduling of stabilizing control tasks," IEEE Trans. on Automatic Control, vol. 52, no. 9, pp. 1680$1685,2007$.

[12] M. Mamduhi, F. Deroo, and S. Hirche, "Event-based data scheduling for a class of interconnected networked control systems," in 54th IEEE Conference on Decision and Control (CDC), pp. 4183-4189, 2015.

[13] J. Wu, Q. S. Jia, K. H. Johansson, and L. Shi, "Event-based sensor data scheduling: Trade-off between communication rate and estimation quality," IEEE Trans. on Automatic Control, vol. 58, no. 4, pp. 10411046, 2013.

[14] M. H. Mamduhi and S. Hirche, "Try-once-discard scheduling for stochastic networked control systems," International Journal of Control, pp. 1-15, 2018.

[15] N. Bisnik and A. A. Abouzeid, "Queuing delay and achievable throughput in random access wireless ad hoc networks," in 3rd Annual IEEE Communications Society on Sensor and Ad Hoc Communications and Networks, vol. 3, pp. 874-880, 2006.

[16] A. Eryilmaz and R. Srikant, "Fair resource allocation in wireless networks using queue-length-based scheduling and congestion control," IEEE/ACM Trans. on Networking, vol. 15, no. 6, pp. 1333-1344, 2007.

[17] L. Huang, S. Moeller, M. J. Neely, and B. Krishnamachari, "LIFObackpressure achieves near-optimal utility-delay tradeoff," IEEE/ACM Transactions on Networking, vol. 21, no. 3, pp. 831-844, 2013.

[18] C. T. Ee and R. Bajcsy, "Congestion control and fairness for many-toone routing in sensor networks," in Proceedings of the 2Nd International Conference on Embedded Networked Sensor Systems, SenSys '04, (New York, NY, USA), pp. 148-161, ACM, 2004.

[19] V. J. Venkataramanan, X. Lin, L. Ying, and S. Shakkottai, "On scheduling for minimizing end-to-end buffer usage over multihop wireless networks," in Proceedings IEEE INFOCOM, pp. 1-9, 2010.

[20] B. W. Carabelli, R. Blind, F. Dürr, and K. Rothermel, "State-dependent priority scheduling for networked control systems," in 2017 American Control Conference (ACC), pp. 1003-1010, 2017.

[21] H. Chan and U. Özgüner, "Closed-loop control of systems over a communications network with queues," International Journal of Control, vol. 62, no. 3, pp. 493-510, 1995.

[22] A. Haghighi and D. Mishev, Delayed and Network Queues. Series in Operations Research and Management Science Series, Wiley, 2016. 